\title{
Measuring and modeling optical diffraction from subwavelength features
}

\author{
Xu Wang \\ California Institute of Technology, Pasadena, California 91125 \\ Jeffrey Mason, Milton Latta, and Timothy C. Strand \\ IBM Almaden Research Center, San Jose, California 95120 \\ David Marx \\ Dynamics Technology, Inc., Torrance, California 90503 \\ Demetri Psaltis \\ California Institute of Technology, Pasadena, California 91125
}

Received January 5, 2000; revised manuscript received August 8, 2000; accepted August 21, 2000

\begin{abstract}
We describe a technique for studying scattering from subwavelength features. A simple scatterometer was developed to measure the scattering from the single-submicrometer, subwavelength features generated with a focused ion beam system. A model that can describe diffraction from subwavelength features with arbitrary profiles is also presented and shown to agree quite well with the experimental measurements. The model is used to demonstrate ways in which the aspect ratios of subwavelength ridges and trenches can be obtained from scattering data and how ridges can be distinguished from trenches over a wide range of aspect ratios. We show that some earlier results of studies on distinguishing pits from particles do not extend to low-aspectratio features. (C) 2001 Optical Society of America

OCIS codes: $050.1960,120.4630,120.5820,290.5880$.
\end{abstract}

\section{INTRODUCTION}

Optical scattering from subwavelength features is getting ever-increasing attention, driven by its importance to a number of technology areas. Included in these are optical storage $^{1-4}$; optical inspection, particularly in the semiconductor industries ${ }^{5,6}$; digital optics ${ }^{7}$; and metrology. 8,9 In all of these areas we are dealing with submicrometer features and often also with dimensions that are small in comparison to the wavelength of the optical sources involved. In the inspection arena we are specifically interested in scattering from isolated subwavelength features on smooth, flat substrates. Much of the previous work in this area was driven by semiconductor wafer inspection, where the main defects tend to be particles on the surface or pits in the surface. Experimental work has concentrated on using single-size particles of known size to dust a surface. ${ }^{10}$ This has two experimental advantages: The defects are well characterized without having to characterize individual particles, and the density of particles is generally set so that there are always several (identical) scatterers in the beam at any time. The high density of particles eliminates the need to search for a specific defect and increases the signal strengths. Modeling of scattering from spheres or ellipsoids on a substrate also has the advantage of being more tractable than the case of a generalized scatterer..$^{5}$

We have chosen to look at single isolated features. For our experimental work the features are made by a focused ion beam (FIB) process, which allows us to make features of different sizes and shapes down to the submicrometer range. The FIB process is very flexible in that it can etch features into a surface or deposit material onto a surface. It can also be used to simultaneously create reference marks that later help in locating the features for the scattering experiments. Modeling of FIB-produced features requires the ability to handle features with arbitrary cross sections rather than being specific to spheres or some other specific shape. The model used in this paper is restricted to two-dimensional features, but the method is applicable to arbitrary profiles. We present the results here of modeling and experimental work on scattering from isolated features of varying shapes and of lateral dimensions ranging from 180 to $840 \mathrm{~nm}$. After demonstrating excellent agreement between the experimental measurements and the model, we use the model to answer some questions about what information can be obtained about subwavelength features from their scattering characteristics. We show, among other things, that a commonly used technique for distinguishing bumps from pits that was developed by use of the spherical particle model does not extend to low-aspect-ratio features. We also show two different ways in which the feature aspect ratio, or the ratio of feature height to width, can be extracted from scattering data on trenches or ridges. 
Our modeling work and experimental work focus on two-dimensional features, i.e., features such as trenches and ridges that are essentially independent of one axis in the scattering plane. Some of the results can be extended directly to three-dimensional features, while others are specific to two dimensions. The next section describes our samples, experimental setup, and measurement process. After that we discuss the background and special aspects of the modeling work. Then we compare the modeling and experimental results. Next, we describe the use of the model to answer some questions that are not easily replicated in the experimental work. Finally, we present the conclusions of our work.

\section{MEASURING DIFFRACTION FROM SUBWAVELENGTH FEATURES}

A. Sample Fabrication and Characterization

Starting with a polished $\mathrm{Al}$ substrate plated with a 10$\mu \mathrm{m}$-thick layer of $\mathrm{NiP}$, we fabricated a series of subwavelength features in the NiP layer by using a Micrion 9500 FIB system. A 50-nm aperture, a beam current of approximately $50 \mathrm{pA}$, and doses in the range $0.1-0.5 \mathrm{nC} / \mathrm{cm}^{2}$ were used to mill away material and create uniform linear trenches with well-defined dimensions. These trenches were designed to be $100 \mu \mathrm{m}$ long with varying widths and depths. They were fabricated in a linear array with a spacing of $150 \mu \mathrm{m}$ between adjacent trenches. Reference marks were also milled into the NiP so that the trenches could be easily located during subsequent measurements.

The topography of each trench was measured with a Digital Instruments Dimension 5000 Atomic Force Microscope (AFM). Standard Si cantilevers with integrated single-crystal Si tips were used in the intermittent contact mode (TappingMode) for height measurements. The nominal tip radius of curvature was $5-10 \mathrm{~nm}$. Data were acquired over a $5 \mu \mathrm{m} \times 5 \mu \mathrm{m}$ area and averaged along each trench. The cross-sectional analysis results are shown in Fig. 1 and summarized in Table 1. In addition, the root-mean-square roughness of the NiP surface adjacent to the trenches was measured to be $0.5 \mathrm{~nm}$.

With a Woollam M44 ellipsometer, the index of refraction of the NiP layer was measured to be 2.07-2.78i for incident light with a wavelength of $633 \mathrm{~nm}$. Ellipsomet-

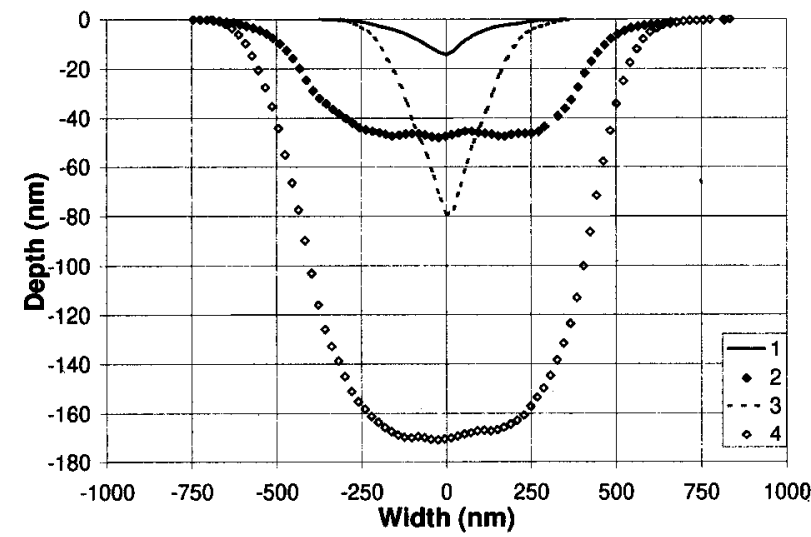

Fig. 1. Average AFM cross sections of FIB-produced trenches.
Table 1. Summary of AFM Data for FIB-Produced Trenches $^{a}$

\begin{tabular}{lccc}
\hline & Width (nm) & Depth (nm) & $\begin{array}{c}\text { Cross-Sectional } \\
\text { Area }\left(\mathrm{nm}^{2}\right)\end{array}$ \\
\hline Profile 1 & 180 & 15 & 3,100 \\
Profile 2 & 800 & 47 & 38,900 \\
Profile 3 & 210 & 80 & 18,200 \\
Profile 4 & 840 & 170 & 142,200 \\
\hline
\end{tabular}

${ }^{a}$ The width is the full width at half maximum (FWHM). The depth is measured from the surrounding surface level to the deepest point of the trench. The cross-sectional area is calculated by integration of the curves in Fig. 1.

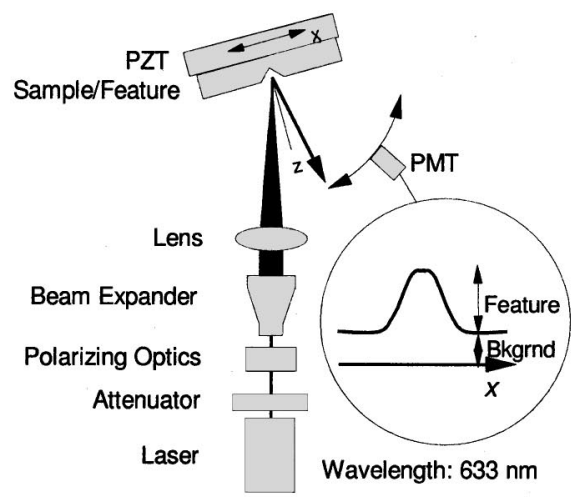

(a)

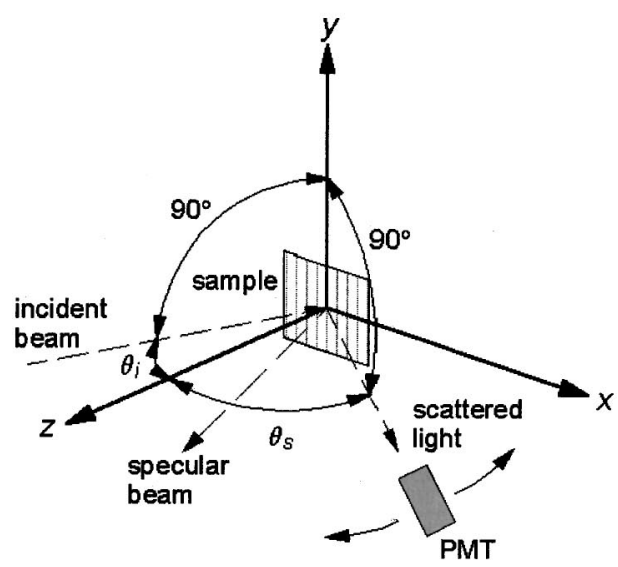

(b)

Fig. 2. (a) Schematic diagram of the experimental setup used to measure the diffracted far field from subwavelength linear trenches. (b) Coordinate system for the far-field measurements. The trenches are aligned along the $y$ axis, and the PMT rotates about the $y$ axis in the $x-z$ plane.

ric data was collected over a range of wavelengths from 500 to $800 \mathrm{~nm}$ with a fixed incident angle of $65^{\circ}$ and analyzed with the WVASE software package provided with the Woollam ellipsometer. The index of refraction was determined by fitting the ellipsometric data with a Lorentz Oscillator model. This index data and the AFM profile measurements are used as inputs to the numerical model described in Section 3.

\section{B. Scatterometer System}

To measure the optical scattering from our subwavelength features, we set up a system as in Fig. 2(a). The 
uniform linear trenches scatter light in only one dimension, so we designed the system to make one-dimensional measurements.

A $10-\mathrm{mW}$ HeNe laser with a wavelength of $632.8 \mathrm{~nm}$ provides a Gaussian beam with a full width at half maximum (FWHM) of $0.48 \mathrm{~mm}$. Neutral density filters in combination with a liquid-crystal variable retarder and polarizing beam splitter (PBS) allow fine tuning of the laser power over a wide range of powers, from $10 \mathrm{~mW}$ down to $10 \mathrm{nW}$. The PBS and a removable half-wave plate control the polarization of light incident on the samples so that the $\mathbf{E}$ field is either parallel to the plane of incidence ( $p$ polarized) or perpendicular to the plane of incidence $(s$ polarized). The PBS transmits $p$ polarized light with an extinction ratio of 1600:1. The half-wave plate can be inserted into the beam downstream from the PBS to rotate the polarization through an arbitrary angle. In these experiments the half-wave plate is oriented to transmit $s$ polarized light with an extinction ratio of 1300:1. Next, a two-lens telescope expands the beam by a factor of 5.9 so that the final focusing lens $(f=50.8 \mathrm{~mm})$ produces a focused spot with a theoretical FWHM of $5 \mu \mathrm{m}$ at the sample surface. Using a Photon, Inc., BeamScan Laser Beam Profiler (Model 2180), we measured the FWHM of the focused spot to be $6 \mu \mathrm{m}$.

To facilitate the description of how we orient our samples, we define a rectangular coordinate system that has its origin at the point where the incident beam comes to a focused spot at the sample surface, as shown in Fig. 2(b). We call the intersection of the plane of incidence with the sample surface the $x$ axis, and we call the surface normal the $z$ axis. Our features, the FIB-produced trenches described above, are oriented with their long axis along the $y$ axis normal to the plane of incidence for all of the experiments described here. For nonnormal incidence, the polarization of the incident beam is described with the normal conventions for $s$ and $p$ polarizations described above. Because of the nature of the feature, it is useful to distinguish between polarization aligned with the long axis of the feature or perpendicular to it, even for normal incidence. We use a notation such that an $s$ polarization vector is always parallel to the $y$ axis, which is also the long axis of the features, and $p$ polarization is perpendicular to the feature axis [see Fig. 2(b)].

The sample is mounted on a piezoelectrically driven (PZT) stage with a range of motion of $80 \mu \mathrm{m}$ in the $x$ direction so that the trenches can be scanned back and forth through the beam. The PZT stage is mounted on an $x-y$ stage so that the sample can be moved in the $x-y$ plane to locate different trenches on the sample surface. In addition, the sample can be independently rotated about the $z$ axis to orient the trenches along the $y$ direction so that they are normal to the PZT motion.

A Hamamatsu H5784 photomultiplier tube (PMT) is mounted $85 \mathrm{~mm}$ from the sample on a rotary stage that independently rotates about the $y$ axis so that the detector can scan through a range of scattering angles $\theta_{s}$. In this setup the detector, the sample normal, and the plane of incidence all lie in the $x-z$ plane. A narrow slit that is $1 \mathrm{~mm}$ wide and $10 \mathrm{~mm}$ high, oriented in the $y$ direction, and bisected by the $x-z$ plane is mounted on the PMT stage $12 \mathrm{~mm}$ in front of the detector face so that only a thin band of light is collected for each $\theta_{s}$. The slit is covered with diffusing material to spread this thin band of light more evenly over the detector face. The dimensions of the slit allow the PMT to collect all of the light in the $y$ direction over a small region $\Delta \theta_{s}=0.81^{\circ}$ about $\theta_{s}$. This collection geometry reduces our scattering measurements to one dimension, which facilitates comparison with the model described below in Section 3. In addition to the slit and diffuser, we have also mounted a laser line bandpass filter with a FWHM pass band of $10 \mathrm{~nm}$ in front of the detector face to suppress ambient light. To minimize multiple reflections between the filter and the sample, we have oriented the filter so that the less-reflective side faces the sample.

The angle of incidence $\theta_{i}$ is changed by rotating the sample (along with the PZT stage and the $x-y$ stage to which the sample is mounted) about the $y$ axis. For these experiments we use $\theta_{i}=5^{\circ}$ and $\theta_{i}=15^{\circ}$. Using $\theta_{i}=5^{\circ}$, we can easily collect data on both sides of the specular beam without worrying about laser feedback from the specular beam reentering the laser cavity. However, with this geometry we cannot measure the specular beam itself, because the detector blocks the incident beam in this region. To measure the specular region, we must increase the angle of incidence. Arbitrarily, we choose $\theta_{i}=15^{\circ}$.

\section{Scattering Measurements}

To measure optical diffraction from our FIB-produced trenches, we focus the incident beam onto a single trench and rotate the PMT about the $y$ axis, as in Fig. 2. In this way we are able to measure the light irradiating the detector at different spatial frequencies $k$, where

$$
k=\frac{\sin \theta_{s}-\sin \theta_{i}}{\lambda} .
$$

This spatial-frequency- $k$ space may be divided into two distinct regions: (1) the specular region, in which the specular beam makes a significant contribution to the measured irradiance, and (2) the scattering region, in which the specular beam makes a negligible contribution to the measured irradiance. We make this distinction because we use a different measurement technique for each region. In our experiments we have defined the transition between these two regions to be at $|k|$ $=0.2 \mu \mathrm{m}^{-1}$.

For $|k|<0.2 \mu \mathrm{m}^{-1}$, we adjust the $x-y$ stage so that the incident beam is centered on a stationary trench, and we measure the light irradiating the detector. For $|k|$ $>0.2 \mu \mathrm{m}^{-1}$, we oscillate the trench back and forth through the focused laser spot by using the PZT stage in order to distinguish background light from diffracted light. When the trench is not under the spot, the PMT detects only the tail of the specular beam and background light such as that due to surface roughness, ambient light, and scattering from optical components. When the trench is centered on the spot, however, the PMT detects a maximum in scattered light that is due to diffraction from the feature. So, as the trench is scanned through the beam, the PMT detects a peak on top of a flat background, as shown in the schematic plot of scattered light 
in Fig. 2(a). We define the scattered light in this region as the difference between the peak and the background, which allows us to measure weak diffracted light signals in the presence of unwanted background light.

Because the measured irradiance falls so quickly in the region $|k|<0.2 \mu \mathrm{m}^{-1}$, we rotate the PMT about the $y$ axis in relatively small increments of $0.25^{\circ}$. In the region $|k|>0.2 \mu \mathrm{m}^{-1}$, the irradiance changes more slowly, and we rotate the PMT about the $y$ axis in larger increments of $2.5^{\circ}$.

To ensure that the light irradiating the PMT remains within the detector's optimal range, we collect data in sets with different incident laser powers that are appropriate for different regions of $k$ space. To measure the incidence power used in a particular data set, we place a Newport optical meter (Model 2835-C) in the beam between the beam expander and the final focusing lens. All scattering readings are normalized to the incident power.

There are two primary sources of error in these experiments. The first involves the accuracy with which we can center a stationary feature beneath the beam when we collect data in the region $|k|<0.2 \mu \mathrm{m}^{-1}$. This error is negligible at $k=0$ but can be as much as $15 \%$ at $k$ $=0.2 \mu \mathrm{m}^{-1}$. This error does not affect the data for $|k|$ $>0.2 \mu \mathrm{m}^{-1}$ because of the method used for data collection. The second involves the nonuniform width of the collection aperture used in front of the PMT. This is a multiplicative error in the normalization that affects all the data uniformly. The amount of this error is less than $7 \%$.

\section{MODELING DIFFRACTION FROM SUBWAVELENGTH FEATURES}

The modeling work described here is based on a numerical model that was developed previously ${ }^{2}$ to predict the optical diffraction of subwavelength structures at a focused spot by the integral method. ${ }^{11}$ The essential idea is to use the surface current concept to solve Maxwell's equations with the claim that the electric and magnetic field everywhere can be calculated from the tangential surface current in a source free domain. For the current work we are concentrating more on the application of the program rather than the modeling itself. Nevertheless, we modified some aspects of the model as described below.

\section{A. Incident Beam}

In previous modeling, for simplicity, the incident beam was defined as a truncated rectangular shape, where the complex amplitude distribution of the electric field TE at the front focal plane of the lens $B(u)$ was given by

$$
B(u)= \begin{cases}1 & \text { if } \quad u<\mathrm{NA} \\ 0 & \text { if } u>\mathrm{NA}^{\prime}\end{cases}
$$

where $u$ is the coordinate at the front focal plane of the focusing lens and NA corresponds to the numerical aperture of the focusing lens. Owing to this definition's sharp transition at the edge of the beam, the corresponding farfield intensity distribution usually shows a strong overshoot at the beam edges. Since in our experiment we used laser beams with Gaussian distribution profiles, a

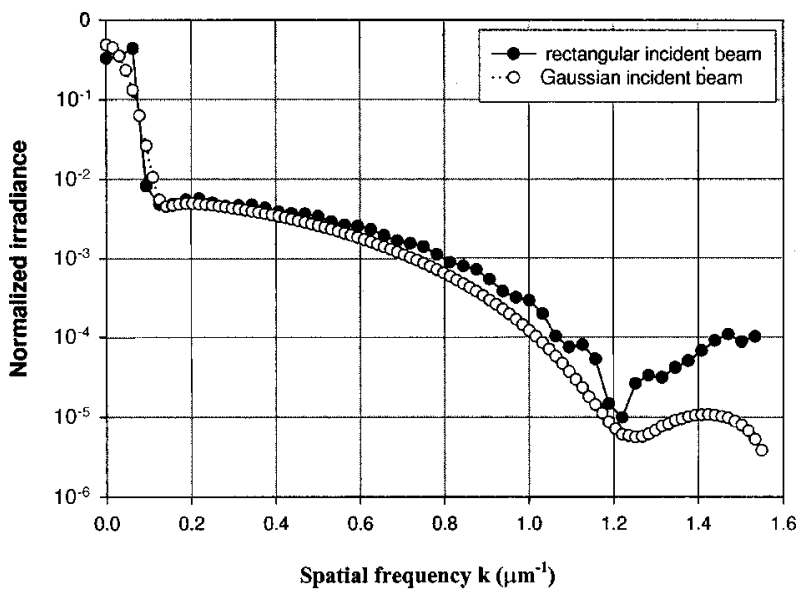

Fig. 3. Far-field diffraction comparison of rectangular versus Gaussian-shaped incident beams.

more natural and more accurate way to represent the incident beam is with a Gaussian profile. Here we define the optical beam intensity to be reduced to 1/e of its peak value at the boundary of the focusing lens. The expression for the incident beam is then given by

$$
B(u)=\exp \left(-u^{2} / \mathrm{NA}^{2}\right) .
$$

To model nonnormal-incidence angles, the illumination beam profile is modified by shifting the incident field at the front focal plane of the lens by $\sin \left(\theta_{i}\right)$, where $\theta_{i}$ is the angle of incidence:

$$
B(u)=\exp \left[-\left(u-\sin \theta_{i}\right)^{2} / \mathrm{NA}^{2}\right] .
$$

Figure 3 shows the calculated far-field diffraction comparison between the rectangular-shaped incident beam and the Gaussian-shaped incident beam for the same sample (profile 2). It clearly shows that the far-field distribution from the Gaussian-profile incident beam has smoother behavior than that from the rectangular incident beam.

\section{B. Sample Profiles}

As previously described, we have made several samples for our comparison of modeling with measurement. The surface contour used in the modeling is defined by AFM measurements, as described in Section 2.A. Our software requires an analytical function for the surface contours so that the necessary sampling points as well as the spacing along the contour can always be computed. We have developed a subroutine and integrated it with the original software package to create a contour $Z(\mathrm{x})$ by Spline interpolating the AFM measurement data (x_data[0...N-1]\&z_data[0...N-1], where $N$ is the number of measurement points). This subroutine also forces the first derivative of the vertical height function to be zero at the boundaries of the measurement range. This guarantees that the generated contour is singularity free within the whole region.

\section{COMPARING MEASUREMENT AND MODELING RESULTS}

We confirm the validity of the model by comparing it with measured scattering data. We show two sets of data for 
comparison; one is with the beam incident at $15^{\circ}$ to the sample normal, and the other is with the beam incident at $5^{\circ}$ to the sample normal. As is explained in Section 2.C, the data are plotted versus spatial frequency.

In Figs. 4-6, both the measured and the modeled irradiance results have been normalized so that the peak irradiance of the incident beam is unity. Accordingly, the peak irradiance of the specular beam for an unmodulated mirror surface is simply the reflectance $R$, as in Fig. 4 at $k=0$. It is also apparent from Fig. 4 that the measured irradiance is Gaussian over more than 2 orders of magnitude. However, at low light levels the measured irradi-

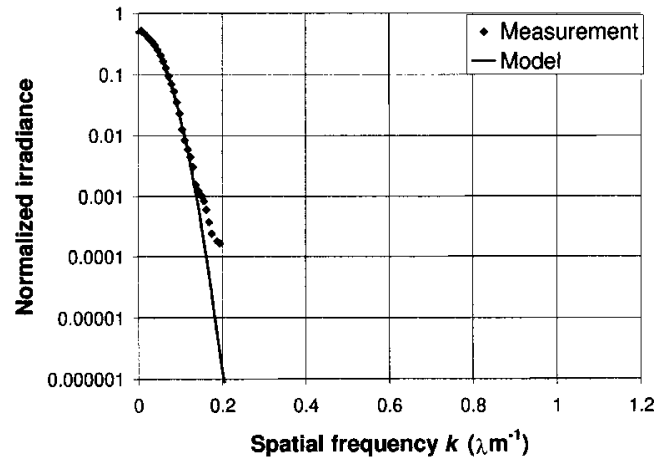

Fig. 4. Specular beam with no feature present, $p$ polarization. $\theta_{i}=15^{\circ}$.

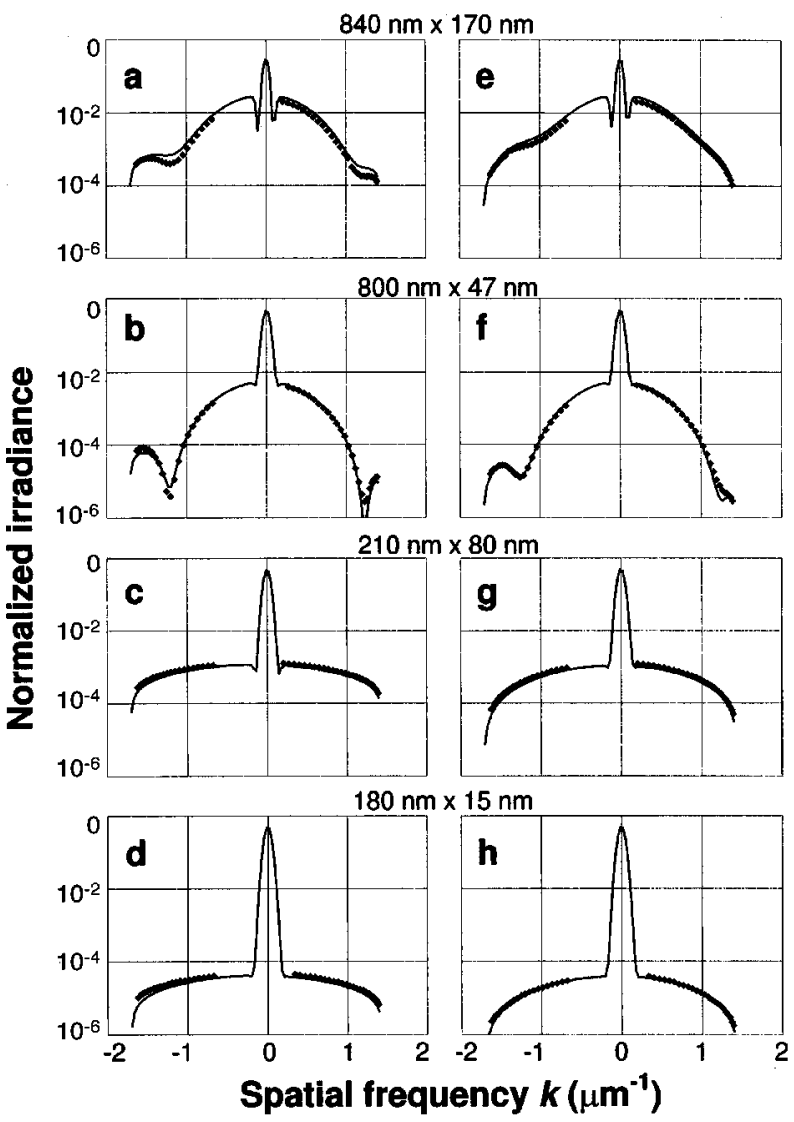

Fig. 5. Modeled and experimental scattering data from four trenches. Figures a-d are for $p$ polarization and e-h are the corresponding plots for $s$ polarization. The incidence angle is $5^{\circ}$ for all cases. The solid curve is the modeling result, and the diamonds are the experimental data.
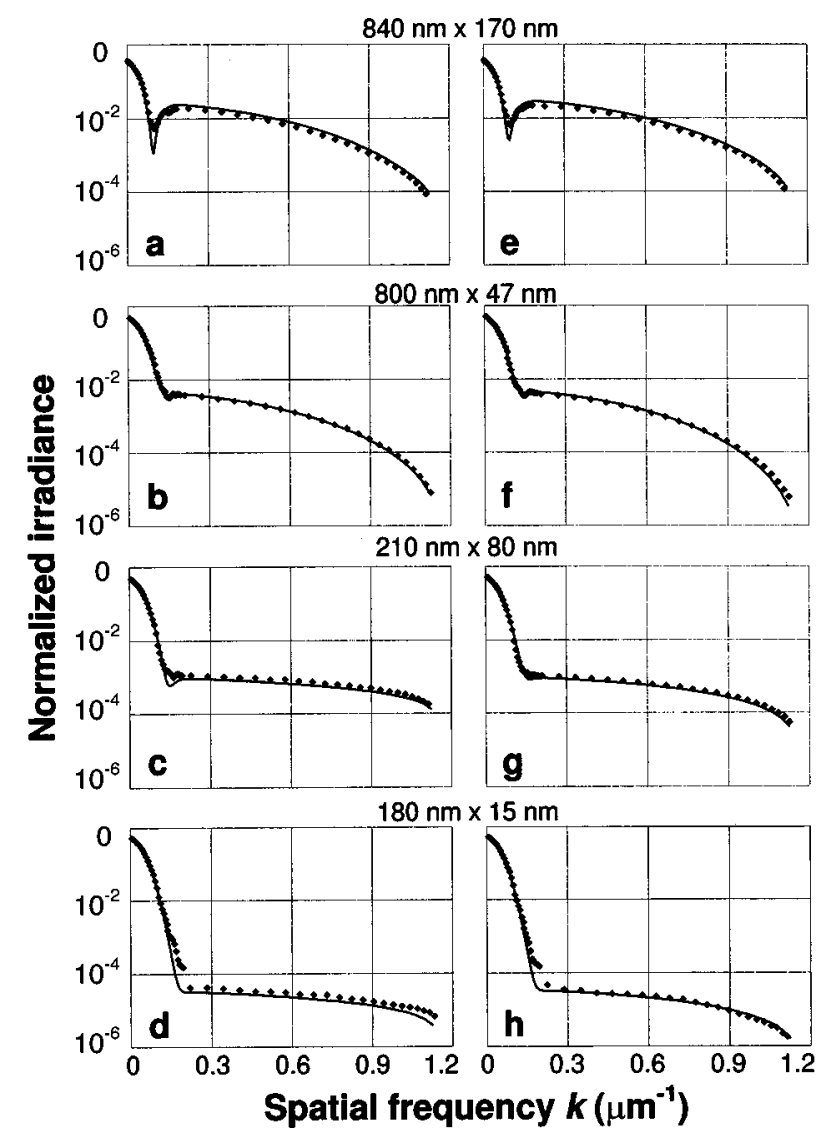

Fig. 6. Same as Fig. 5 for a $15^{\circ}$ angle of incidence. Figures a-d are for $p$ polarization and e-h are the corresponding plots for $s$ polarization.

ance becomes non-Gaussian and higher than the model's prediction. This is primarily due to additional light coming from multiple reflections between the sample and the detector. This same non-Gaussian behavior is evident in Figs. 6(d) and 6(h), since the scattered light is more than 4 orders of magnitude weaker than the specular peak. Multiple reflections and other sources of background light do not affect the measured irradiance in the region $k$ $>0.2 \mu \mathrm{m}^{-1}$, because they are subtracted by the data collection technique used in this region, as described in Section 2.C.

Overall, the excellent agreement (in Figs. 5 and 6) between the model predictions and the actual measured scattering gives us confidence in the validity of the model. It works well throughout the critical transition region from features larger than the wavelength to subwavelength structures.

With $\theta_{i}=5^{\circ}$, the scattering shows a strong dependence on scattering angle for features larger than the wavelength (see Figs. 5(a), 5(b), 5(e), and 5(f)). Especially for $p$ polarization there is a minimum at a large scattering angle. The origin of this high-angle minimum is the destructive interference of the two scattered fields from the contour edges of the trench. For example, in Fig. 5(b), this high-angle minimum is obtained at $k$ $=1.2 \mu \mathrm{m}^{-1}$. This corresponds to two point scatterers separated approximately by $370 \mathrm{~nm}$. Given that the trench in Fig. 5(b) has a FWHM width of $800 \mathrm{~nm}$ and an approximately $400-\mathrm{nm}$ width at the bottom, it appears 
that this high-angle minimum occurs because of the scattering from the edges of the trench bottom for $p$ polarization. Similar behavior is observed in Fig. 5(a) for the trench with a deeper profile. However, this minimum at a large scattering angle is not observed for subwavelength trenches and is less pronounced for $s$ polarization. Nevertheless, this minimum can be used to extract contour information about the larger trenches.

In Figs. 5(a) and 5(e) the model predicts another type of minimum at a smaller scattering angle $(k$ $\left.=0.15 \mathrm{\mu m}^{-1}\right)$. The experimental setup did not permit measurements in this range with $\theta_{i}=5^{\circ}$. To study this low-angle minimum further we made a new set of measurements by rotating the sample to $\theta_{i}=15^{\circ}$. The results are shown in Fig. 6. This low-angle minimum is due to the interference between the specular beam and the scattered light. In other words, the reflected field from the top flat surface interferes with the reflected field from the trench contour. If the trench depth is approximately $\lambda / 4$, then the reflected and scattered fields are out of phase with each other. In general, the scattered light has a broader angular distribution than the specular reflection. Therefore, at small angles, the scattered field measured in the far field is dominated by the specular reflection, whereas the reverse is true for large scattering angles. There exists an intermediate angle at which the two contributions are of equal strength but out of phase, and they destructively interfere. This gives rise to the low-angle minimum that is obtainable for subwavelength trenches as well. In particular, Figs. 6(c) ( $p$ polarization) and $6(\mathrm{~g})(s$ polarization) show the scattering data from the trench that is $210 \mathrm{~nm}$ FWHM and $80 \mathrm{~nm}$ deep. We observed a low-angle minimum only for the $p$ polarization, because the walls of the trench behave similar to a planar waveguide that allows the $p$ polarization to penetrate to the bottom and reflect back. The $s$ polarization can only penetrate a waveguide with feature size of approximately half wavelength. ${ }^{12}$ Therefore, in $s$ polarization, this type of minimum is observed only for the larger trenches [Figs. 6(e) and 6(f)]. This low-angle minimum is particularly useful for characterizing subwavelength features, since we can define the direction of a subwavelength scratch by comparing the $s$ and $p$ polarization scattered fields, and the depth of the trench can be estimated from the magnitude of the measured minimum. More details about this are described in the next section.

\section{EXTENDED MODELING RESULTS}

Having established the agreement between modeling and experiment, we can use the model to investigate questions that are not easily studied experimentally. For example, although the scattering information can be used to obtain some size and shape information about features that are larger than the interrogating illumination wavelength, the amount of information obtainable for subwavelength features is much more limited. Here we ask, using our model, if it is possible to determine the aspect ratio of subwavelength features and differentiate features extending above the surface from those that extend into the surface, e.g., ridges versus trenches. For this study four different trenches, all having the same cross- sectional area of $10,000 \mathrm{~nm}^{2}$ but with different aspect ratios, are proposed; the cross sections are shown in Fig. 7.

A fundamental piece of information about a feature on a surface is whether it is sticking up from the surface or down into the surface. This is, for instance, important in semiconductor wafer inspection, where you want to distinguish between particles on the surface and pits in the surface. For that application considerable experimental and theoretical work was done in which the particles were modeled by spheres and ellipsoids., ${ }^{5,6}$ On the basis of this work, a common technique has been adopted by many vendors of inspection equipment that relies on using oblique-incidence $p$ polarization for illumination. It has been found that the forward and the backward scattering contributions from a sphere on the surface are relatively stronger than the scattering normal to the surface. For a pit, the opposite is true. Thus, with two detectors used to monitor forward and backward scattering and with ratioing their signal to that of a third detector monitoring light scattered normal to the surface, particles can be distinguished from pits. The modeling results in Fig. 8(a) show this difference for a trench versus a ridge with an aspect ratio of approximately $1: 1$, i.e., an aspect ratio similar to that of a sphere. The ridges were formed by our taking the same profiles as in Fig. 7 but inverting them. The beam is $p$ polarized and incident at $60^{\circ}$. For the ridge, the dip in scattering normal to the surface compared with forward and backward scattering is clear. For the trench, the dip is not there, confirming the earlier work for spheres versus pits. However, as the aspect ratio decreases, this dip shifts toward the specular beam and eventually disappears. Figure 8(b) shows the case of a feature with an aspect ratio of approximately 1:4. Here the trench and the corresponding ridge are indistinguishable. Such low-aspect-ratio features are common in inspection of computer hard disks. Reference 5 indicates a similar disappearance of the $\operatorname{dip}$ in $p$ scattering in the surface normal direction for an oblate spheroid with a major-to-minor axis ratio of 1:1.5.

Figures 9(a) and 9(b) show the scattering patterns for normal-incidence light with the polarization perpendicular to the trench ( $p$ polarization) and parallel to the

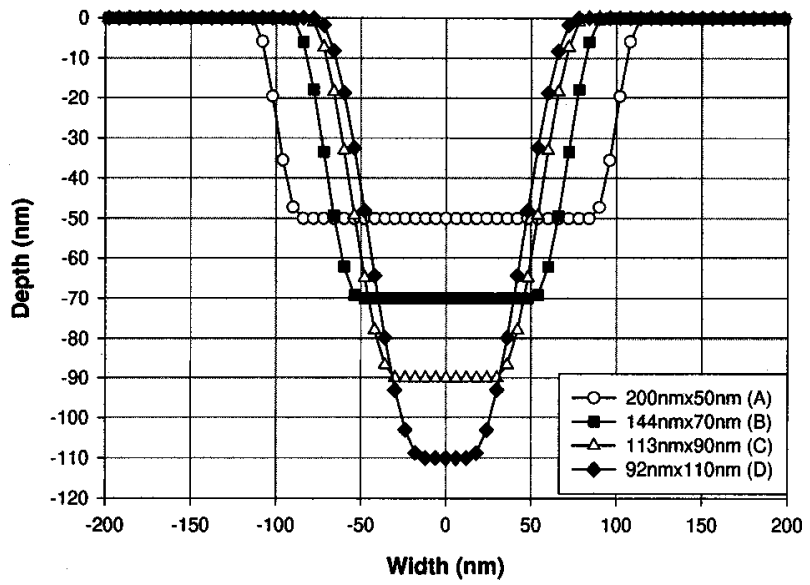

Fig. 7. Cross-sectional profiles of four different trenches, all having the same cross-sectional area but different aspect ratios. The aspect ratios range from approximately 1:4 (depth to width) to approximately $1: 1$. 


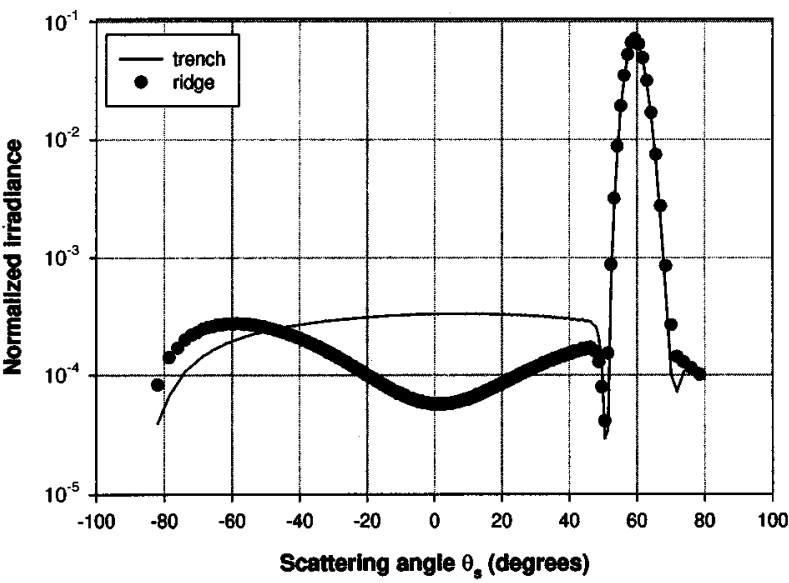

(a)

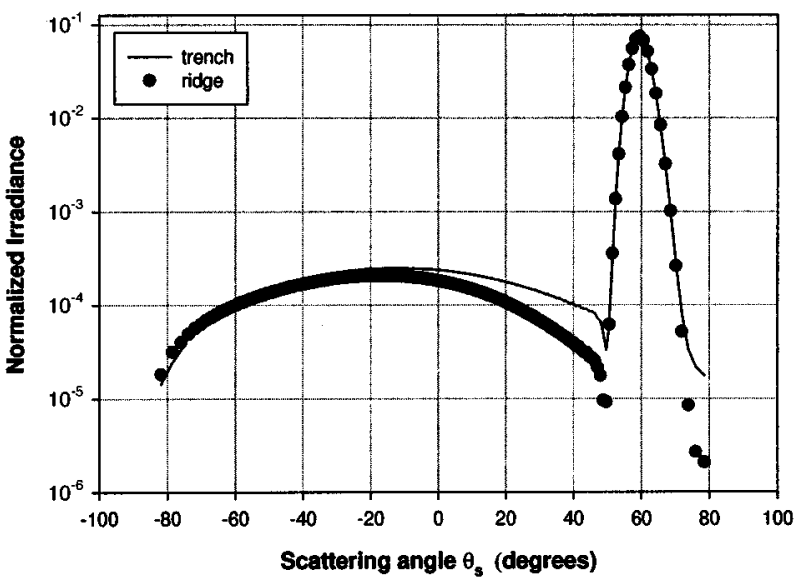

(b)

Fig. 8. Scattering for trenches versus ridges. The illumination is at $60^{\circ}$, and is $p$ polarized: (a) for feature with an aspect ratio of 1:1, (b) for feature with an aspect ratio of 1:4 (depth to width).

trench ( $s$ polarization), respectively. The $p$ polarization case shows that the scattering is essentially indistinguishable for the four different features except in the region where the low-angle minimum occurs. However, the $s$ polarization case shows a level shift in irradiance between the different aspect-ratio cases. Figure 10 shows the ratio of the $s$ and $p$ polarization scattering intensity, measured at two fixed scattering angles, i.e., $11^{\circ}$ and $40^{\circ}$, for the four different aspect ratios. The same calculation was also done for the ridges. The results in Fig. 10 clearly show that the ratio of the two polarization components of the scattered light can be used not only to determine the aspect ratio over the range considered here but also to distinguish ridges from trenches. Since these results rely explicitly on the ratio of polarization states, which for the normal-incidence case are defined with respect to the two-dimensional features, they do not extend to three-dimensional features such as particles and pits. They are, however, of use for features such as linear scratches, which are well described by the trench-ridge model. We also remind the reader that these results have assumed features much smaller than the wavelength of illumination. This simple behavior does not hold for features larger than the wavelength, where the scattering patterns are more complex, but in that regime other techniques can be used. This size dependence of the $s / p$ ratio is also discussed for spheres in a recent paper. ${ }^{5}$

Figure 9(a) again shows the sharp low-angle minimum in the scattering at the edge of the specular beam for $p$ polarization. If we zoom in on this minimum as shown in Fig. 9(c), we see that the depth of this minimum varies directly with the aspect ratio. This indicates that we could use measurements with $p$ polarization to infer as-

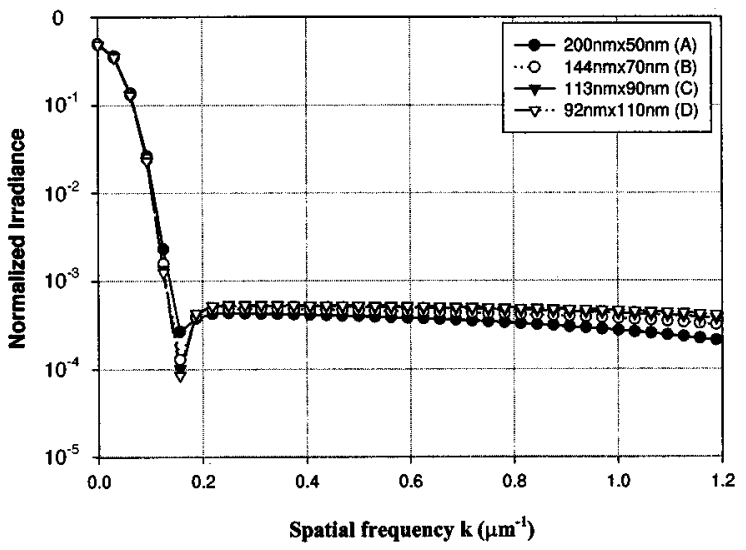

(a)

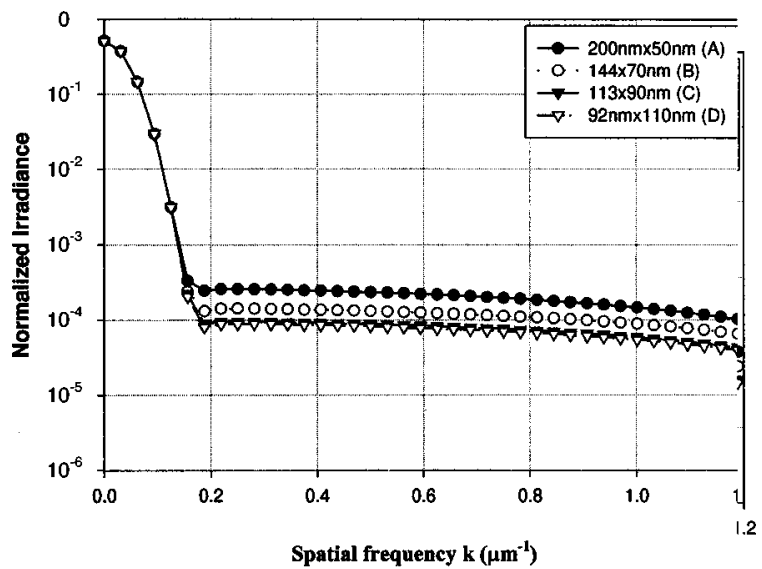

(b)

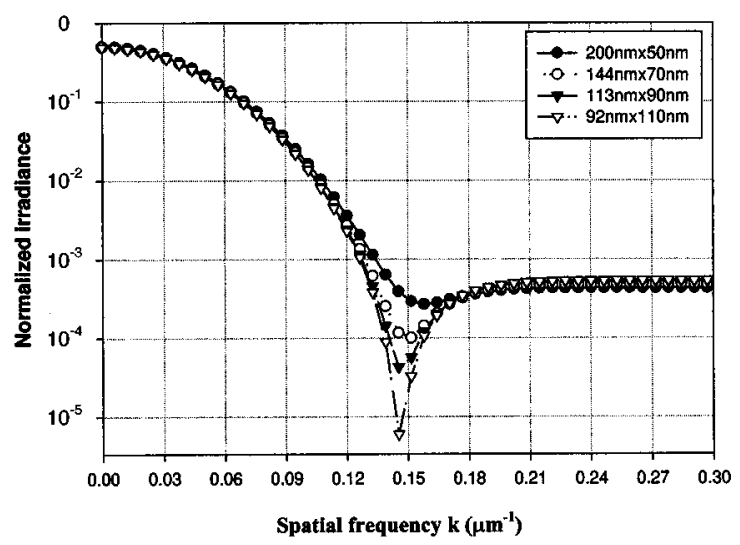

(c)

Fig. 9. Computed scattering from the four trenches of Fig. 7 at normal-incidence illumination. (a) Polarization perpendicular to the trench ( $p$ polarization), (b) polarization parallel to the trench ( $s$ polarization), (c) zooming in on the dip in (a). 


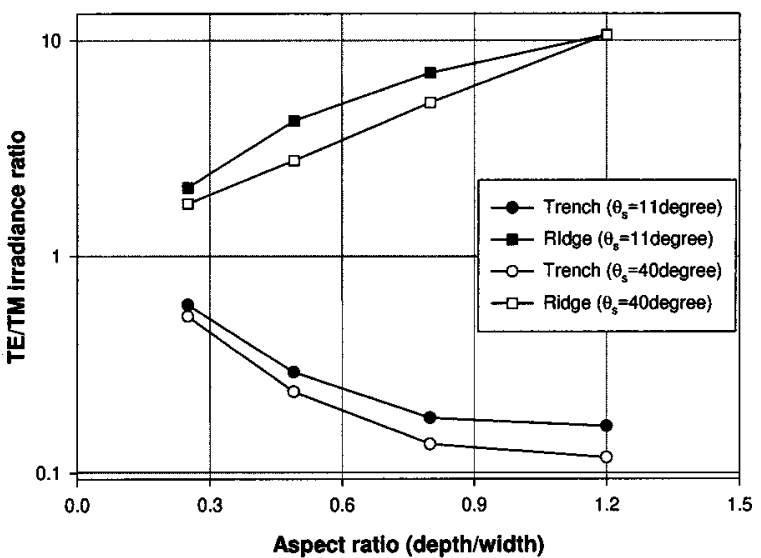

Fig. 10. Ratio of the scattering of light polarized parallel versus perpendicular to the trench axis as a function of the aspect ratio of the feature.

pect ratio by our using the ratio of the scattered intensity at this low-angle minimum to the scattering intensity at a point in the flat portion of the scattering regime, e.g., at a scattering angle of $10^{\circ}$, where $k=0.27 \mu \mathrm{m}^{-1}$.

\section{CONCLUSION}

We have described a simple optical scatterometer for looking at isolated subwavelength features. The features were produced with a focused ion beam process that provided great flexibility in the range of sizes and shapes that could be produced. A numerical modeling program capable of handling arbitrary two-dimensional features in a planar surface was developed. The modeling results were shown to agree very closely with the experimental measurements for scattering from a range of subwavelength features etched into a planar metallic surface. The model was then used to investigate what information could be extracted regarding subwavelength scatterers. We have shown that, for two-dimensional features such as scratches, the effective aspect ratio of the feature can be determined either from the ratio of the scattering from $s$ and $p$ polarized illuminating beams or by monitoring the depth of the minimum in scattering at the edge of the specular beam with only a $p$ polarized illuminating beam. We have also shown that the $s / p$ scattering ratio can be used to distinguish subwavelength trenches from ridges. Finally, we have shown that a commonly used technique for distinguishing subwavelength particles from pits on a surface cannot be extended to features with aspect ratios of 1:4 or smaller. The model developed here can be used to study a wide range of scattering situations for subwavelength features.

\section{ACKNOWLEDGMENT}

This work was supported by the National Science Foundation Engineering Research Center for Neuromorphic Systems Engineering at California Institute of Technology.

\section{REFERENCES}

1. D. S. Marx and D. Psaltis, "Polarization quadrature measurement of subwavelength diffracting structures," Appl. Opt. 36, 6434-6440 (1997).

2. D. S. Marx and D. Psaltis, "Optical diffraction of focused spots and subwavelength structures," J. Opt. Soc. Am. A 14, 1268-1278 (1997).

3. W.-C. Liu and M. W. Kowarz, "Vector diffraction from subwavelength optical disk structures: two-dimensional nearfield profiles," Opt. Express 2, 191-197 (1998), http:llepubs.osa.org/opticsexpress.

4. W. H. Yeh, L. Li, and M. Mansuripur, "Vector diffraction and polarization effects in an optical disk system," Appl. Opt. 37, 6983-6988 (1998).

5. Y. A. Eremin, J. C. Stover, and N. V. Orlov, "Modeling scatter from silicon wafer features based on discrete sources method," Opt. Eng. 38, 1296-1304 (1999).

6. C. A. Scheer, J. C. Stover, and V. I. Ivahknenko, "Comparison of models and measurements of scatter from surface bound particles," in Flatness, Roughness, and Discrete Defects Characterization for Computer Disks, Wafers, and Flat Panel Displays II, J. C. Stover, ed., Proc. SPIE 3275, $102-$ 111 (1998).

7. H. Haidner, D. Dias, L. L. Wang, and T. Tschudi, "Binary subwavelength structures/resonance gratings as polarization elements," Pure Appl. Opt. 7, 1347-1361 (1998).

8. J.-J. Greffet and F.-R. Ladan, "Comparison between theoretical and experimental scattering of an $s$-polarized electromagnetic wave by a two-dimensional obstacle on a surface," J. Opt. Soc. Am. A 8, 1261-1269 (1991).

9. D. Levy, L. Shingher, J. Shamir, and Y. Leviatan, "Step height determination by a focused Gaussian beam," Opt. Eng. 34, 3303-3319 (1995).

10. J. C. Stover and M. Bernt, "A multiple particle technique for determination of differential scattering cross-section of very small surface bound particles," in Optical Scattering in the Optics, Semiconductor, and Computer Disk Industries, J. C. Stover, ed., Proc. SPIE 2541, 108-112 (1995).

11. A. T. De Hoop, Modern Topics in Electromagnetics and Antennas, PPL Conference Publication, Vol. 13, Peter Peregrinus Ltd., Stevenage, England (1977).

12. N. J. Cronin, Microwave and Optical Waveguides (Institute of Physics, London, 1995). 